\title{
Living an Unlived Life Due To Fear of HIV/AIDS: Reflections from Malaysia
}

\author{
Paramaswari Jaganathan. Ph.D.1, Azlinda Azman. Ph.D. ${ }^{2}$ \\ ${ }^{1}$ (School of Languages, Literacies and Translation,Universiti Sains Malaysia) \\ ${ }^{2}$ (School of Social Sciences, Universiti Sains Malaysia)
}

\begin{abstract}
A descriptive qualitative study on the empowerment of People Living with HIV/AIDS in Malaysia revealed that the People Living with HIV (PLHIVs) experience internalised fear that often impacts and protracts the intervention effort carried out by the community-based organisations. The stigmatisation of HIV/AIDS contributed to development of internalised fear amongst PLHIVs. Using the empowerment model of instilling positive living among PLHIVs, we interviewed a total of 25 PLHIVS with a ratio of $60 \%$ male and $40 \%$ female respondents selected from a random sampling. The results showed that the PLHIVs' internalised fear is related to six main factors; painful and discredited death, rejection, disclosure, uncertain future, financial impediments and fear of affecting their loved ones. This paper discusses the fear shared by the PLHIVs through interviews and participation observation sessions. It identifies some of the strategies employed by the community-based organisations in assisting the PLHIV community to manage and overcome their internalised fear to live a positive life. It also highlights some of the lessons learnt in realizing the organisations' effort to empower the PLHIV community in Malaysia.
\end{abstract}

Keywords: community-based organisation, HIV/AIDS, internalized fear, stigma and discrimination, intervention.

\section{Transcending the boundaries of denial syndrome to reality}

In the early years of HIV/AIDS detection in Malaysia, the community-based organizations (CBOs) in Malaysia, were the first to respond to the unmet needs of the persons living with HIV/AIDS [1]. The CBOs provide social support to fulfill the needs of certain marginalised groups particularly the drug users; sex workers and the gay community who were least likely to get help from the government as these groups were initially less recognised in the Malaysian community [1]. In the context of Malaysia too, empowerment is seen as a main avenue to increase self-control and reduce the concept of powerlessness of individual as advocated by Rappaport [2] and Mc Ardle [3]; mainly because the CBOs managing the HIV/AIDS organizations in Malaysia work with limited financial assistance and minimum staffs. Conceptualising on the empowerment model at an individual level, the CBOs in Malaysia initiate effort to impart information, provide social support, increase the selfefficacy of the PLHIVs and motivate them to assume responsibilities for change. These initiatives ultimately are expected to bring out some outcomes; mainly to live life positively, increase their self-efficacy to control on health behaviour, overcome HIV/AIDS stigma and build an active participating community. These outcomes were selected on the basis of its significance and urgency to empower the PLHIVs to have control of their health behaviour.

As noted by Kubler-Ross [4], a person who is diagnosed with a terminal illness, is bound to experience several stages of accepting his status. Similarly, a PLHIV who is detected with HIV infection would experience the denial stage, anger and the bargaining stage as well as the acceptance or the depression stage. For this situation, the CBOs personnel needs to be able to identify the condition of the PLHIVs in order to execute the appropriate intervention initiatives in order to bring about the desired behaviour change in their clients. Page and Nyakanda [5] identified three elements which help PLHIVs to identify and take responsibility for behaviour change. This includes reducing the vulnerability to HIV, promoting good health and preventing diseases. In their research on facilitating community action in HIV/AIDS affected areas in Africa, Page and Nyakanda [5] posited that by taking charge of the behaviour change, the PLHIVs will be able to live life positively as well as increase their self-efficacy. The belief of bringing about change in oneself will then enable the PLHIVs to reject the internalised self-image that they possess as well as transform their dehumanizing experiences into something that is empowering. This belief will then help the PLHIVs to overcome the pre-existing internalised fear and the stigmatisation of HIV/AIDS. With the presence of positive behaviour change and an increase in self-efficacy, the PLHIVs will be able to deal with his surrounding proactively. Studies have showed that the PLHIVs who are able to control their behaviour and have high self-efficacy is less likely to succumb to pressures to conform and he will be able to handle fear more realistically instead of being defensive [4]. Subsequently, the PLHIVs will be able to participate more actively in the community as they are in control of the fear within themselves. 
Coping with HIV/AIDS infection is a long and complex process which begins with confronting internalised fears that evoke in the PLHIVs. Internalised fear or fear which is induced within oneself may vary among the various groups although they may be interconnected. Affected PLHIVs community in Malaysia experience internalised fear mainly due to the stigmatisation of the pandemic and due to the lack of comprehensive knowledge about HIV/AIDS infection, treatment and care. At the onset of HIV/AIDS in Malaysia in the mid-1980s, the debate on HIV-prevention focused on the issues of "core transmitters" particularly the gay community, sex-workers and intravenous drug users. Hence, the social norms and sexual practises which are considered deviant, taboo, abnormal and against the religious mores in the Malaysian society were blamed as the main causes instead of risky sexual behaviours. Some discourses also characterised the AIDS pandemic as a "natural product of modernity's sexual revolution" [6] and "transgression of social norms" [7] These discourses inadvertently contributed to a strong denial syndrome among the Malaysian society until the recent years witnessed a change in the mode of transmission whereby the Malaysian Ministry of Health reported a worrying increase rate of infection amongst women and more children were becoming AIDS orphans in Malaysia [10]. While the stigmatisation of the HIV/AIDS pandemic is closely related to the socio-cultural aspect and religious beliefs of the multi-ethnic society in Malaysia, the lack of knowledge about the pandemic is also a factor that contributes to the internalised fear among the PLHIVs community. The effects of the internalised fear leaves an impact on the self-esteem of the PLHIVs due to the opinion of being seen as "lesser person" and having a "spoiled identity" [8]. Hence, the intervention effort to assist the PLHIVs community, needed to respond to the internalised fear which contributes to denial syndrome and the initial resistance of the PLHIVs to obtain the necessary assistance. The stigmatisation of the HIV/AIDS pandemic, however, impedes the PLHIVs community from obtaining early intervention and treatment. Although systematic involvement of community-based intervention is said to be an effective intervention tool to increase and improve health condition and reduce the powerlessness of individuals in the long run [9];[7] yet a major obstacle that the community-based organisations face is assisting the PLHIV community to address their internalised fear. The internalised fear about HIV/AIDS can be then understood as the preconceived apprehension which is made belief within the PLHIVs as a result of how the society at large constructs it. In the context of Malaysia, the preconceived guiding principle surrounding HIV/AIDS is negative and heavily stigmatised. As such, fear is evoked in the PLHIVs due to the affixed negative perception of HIV/AIDS in the Malaysian setting. In executing intervention initiatives to the PLHIVs, the community-based organisation need to understand and work on the internalised fear among PLHIV community in order to empower the community to live life more positively. The study further identified several issues that contributed to the presence of this fear among the PLHIVs that adversely affects the intervention initiatives of the community based organisations (CBOs).

\section{METHODOLOGY}

This study employed a qualitative study using in-depth interview which was divided into two categories involving the PLHIV community as well as the organisation's personnel. A total of 25 PLHIV community members were interviewed in general to identify their different stages of health condition, their psychosocial needs as well as the various assistance and support obtained from the organisations. The PLHIVs were generally met through hospital visits, home visits, programme involvement and also through introduction by three different organisation involved in the support and care of PLHIVs. The field work covered an estimated period of six months. $60 \%$ of the PLHIVS respondents were male while $40 \%$ were female from the three main races in Malaysia. They included Malay respondents (28\%), Indians (48\%) and Chinese (16\%) as well as East Malaysian and others (8\%). The PLHIVs respondents ranged from 25 years to 65 years old age group. The highest number of respondents (24\%) fell in the 36-40 years age group, followed by 31-35 years age group (20\%) and a similar percentage of $8 \%$ among the $46-50$ years, $56-60$ years and 61-65 years. The respondents also varied in their health status whereby some were compliant to treatment and medication while some were non-compliant to neither medication nor treatment. As for the organisations' personnel, the key informants were interviewed focusing on organisation's role in assisting PLHIVs to cope with their seropositive status and types of social support and networking provided to help empower the PLHIVs. The respondents from the communitybased organisations included EXCO members, outreach programme coordinators, medical officers as well as higher level administrative staffs who were involved directly in the PLHIV programmes. They were the key informants who helped to be the 'resource person' for further explanation when needed.

\section{RESULTS}

A total of 25 PLHIVs were interviewed with consent based on hospital visits, home visits and programme involvement. The respondents were classified according to their health status; PLHIVs who were on treatment (48\%), PLHIVs who are not on HAART treatment but attend follow-up treatment (6\%), PLHIVs who were non-compliant or default treatment (20\%), PLHIVs who were already were in the AIDS stage (12\%) and PLHIVs who were in denial stage and did not seek treatment at all (4\%). The internalised fear among the 
PLHIVs generally encircles issues of discredited death, rejection, disclosure, uncertain future, financial setbacks and fear of affecting the loved ones. These internalised fears were however, coded randomly to identify the types and contributory factors for the presence of the internalised fear.

\section{1: $\quad$ Types of Internalised Fear Faced by PLHIVs in Malaysia Painful death and "inherited discredition"}

The fear of having a painful death is one of the immediate feelings shared by the PLHIVs upon their detection of their seropositive status. A total of $22(88 \%)$ PLHIVs shared their fear of having a painful death. This included two senior citizen PLHIVs who had limited understanding of their HIV status and the infectious disease that had developed as well as another PLHIVs who isolated himself for four years. The PLHIVs fear the pain they would experience before death and had an anxiety over having a "gruesome appearance" upon dying. The fear of pain before death also evoked suicidal thoughts amongst the PLHIVs and caused depression which had led some of the respondents to isolate themselves from relatives and friends. Besides the painful death, the PLHIVs also have an internalised fear of having a discredited death. They fear that the family reputation will be smeared after their death as the cause of death will be known by others. In Malaysia, it is procedural for the health officials or the organisation personnel to be present during the funeral to ensure minimal handling of the body. However, the PLHIVs fear that the presence of the officials will cause suspicion amongst the people who have come to pay their last respect. Moreover, the body that is usually wrapped with a final layer of plastic would cause suspicion among those attending the wake ceremony and needs to be creamated or buried within 24 hours of the death. All these standard precautions evoke suspicion among people who come to pay their last respect. Hence the PLHIV community has the internalised fear of having a discredited death which will eventually victimize their family members or tarnish their family reputation. The family members then will have to bear the "inherited discredition" due to the stigmatisation of HIV/AIDS in Malaysia. The study identified 18 PLHIVs (82\%) who voiced out this element of internalised fear. In-depth interview sessions revealed that the fear of "inherited discretion" was more common among PLHIVs with children and single men as the family reputation was put ahead of their fear of their ailment itself.

\section{Rejection from spouse, family members and relatives}

The internalised fear of rejection from spouse, family and relatives was closely associated to the PLHIVs mode of infection. Spouse rejection was linked to being unfaithful to partner (8\%) and non- disclosure of drug use (4\%); while the family rejection (36\%) was linked to issue of immoral behaviour. Morality is strongly ingrained in the Malaysian culture. As such, immoral behaviour which is associated to prohibited sex and having multiple sex partners tarnishes the family reputation as well. Two respondents (8\%) cited the source of infection as an implication on their visit to commercial sex- workers which in return tarnish the family reputation. Similarly, drug abuse is considered a crime and association to drug abuse also brings shame to the family members. In the Malaysian family institution, upholding the family reputation is an essential responsibility of every family member. Although some of the families were non-judgmental and accepted the seropositive status of the PLHIVs, yet the PLHIVs themselves had formed a presentiment of being rejected by the family and delayed intervention and treatment. They choose not to disclosure their seropositive status in order to avoid rejection from their spouse, family members and relatives due to the stigmatisation of HIV/AIDS in Malaysia.

\section{Sexuality disclosure}

The internalised fear of sexuality disclosure was common among the homosexual PLHIVs and they suffer a double burden of having to disclose their seropositive status as well as their homosexuality to both the medical personnel and their family members. In this study, one respondent (4\%) articulated his status and referred himself as a "mid-night cow-girl" who has multiple partners. Non-disclosure to family members however was linked to the fear of family members' non-acceptance of his sexuality status. The respondent did not want to disappoint his female siblings and parents as he had sustained close family ties with them and was looked up as a loving and caring brother as well as a filial son. The respondent only obtained treatment when his health condition worsened but he was not willing to disclose his homosexuality status. This impedes the process of dispensing appropriate medical treatment as well as obtaining subsequent counseling sessions. The internalised fear leaves great impacts on the homosexual men in making health choices particularly in seeking further treatment and help.

\section{Loss of employment}

More than half of the employed respondents in this study (52\%) experienced internalised fear of losing their employment if their seropositive status is disclosed. Hence, they delay seeking treatment despite knowing of the seropositive status. Generally, they visit the private practitioners to obtain further treatment when they are 
referred for follow-ups or advised by their family members. PLHIVs have reported cases that the medical personnels had notified their seropositive status to the employer although it is against the rights of the PLHIVs. Three cases $(12 \%)$ of employed PLHIVs in this study experienced discrimination whereby they were demoted or transferred to another section upon the detection of the seropositive status. One (4\%) of the respondent was kept under strict supervision of the company nurse. Such discriminatory practices at the workplace had instilled internalised fear among other PLHIVs too. The respondents also feared that the detection of status may cause the loss of employment and affects the opportunity to get promoted in their career. The fear of losing their employment is also drawn from previous incidences that have occurred in the workplace as well as from the neighbouring workplaces.

\section{Loss of support for stay and palliative care}

The loss of support for stay is another factor that invokes the internalised fear amongst the PLHIVs community particularly for the PLHIVs who fear that their disclosure may result in their family members' rejection. PLHIVs that live with their family or in an extended family institution fear the loss of support for stay. A total of eight respondents (32\%) articulated this fear in this study. Hence, two respondents (8\%) refrained the organisation personnel or social workers to visit them to hand out financial aid and services. In Malaysia, there are several shelters and drop-in-centres to cater for the PLHIVs. However, the places are limited and PLHIVs are also skeptical of being placed in the shelters for the fear of being known. Another reason why the PLHIVs have an internalised fear due to loss of support is that they are uncertain of getting continuous help when their condition deteriorates or when they experience physical delibility due to the side effects of the medicine such as the loss of appetite, diarrhoea, fatigue and other complications. The "roller-coaster" effect of medication and treatment may persist for a long time and may change with the treatment regiment substitution. As such, the PLHIVs feel insecure of the livelong care by their family members and caretaker. Hence, they abstain from disclosing their status nor seeking early treatment and care.

\section{Fear of children's uncertain future and "accidental infection".}

Children have become the main concern for married PLHIVs. A total of eight respondents (32\%) expressed their concern over their children's future due to several factors. Firstly, they lacked the information on where to get the support for the children in situations when a death occurs. Secondly, the children themselves are not informed of the parent's seropositive status or the child's own seropositive status. In cases where the PLHIVs do not have family support, the anxiety is higher as the children are not informed of the parent's seropositive status. Likewise, the PLHIVs children are also not informed of their seropositive status as the parents fear their children may disclose it to others. Another observation from this study shows that the children whose parents are incarcerated are not informed of their parents' whereabouts due to the fear that they may be discriminated against. The situation is the same for children whose parents are bed-ridden or have succumbed to AIDS. Generally, the children are told that their parents suffer from terminal illness instead of their seropositive status. In one of the cases, the PLHIVs refused to associate himself with his young nieces and nephews in fear of "possible accidental infections". Such internalised fear was only confronted with dissemination of proper information and counseling.

\section{Fear of financial constraint for treatment and care}

Treatment for HIV is closely linked to the fear of losing employment and loss of family support. Due to the high cost of treatment, T-cell count test or the CD-4 count test as well as the viral load tests, particularly in the private hospitals, many PLHIVs delay their treatment. A total of 11 respondents (44\%) in this study seek further help only when their health condition worsened or when they were admitted in the hospitals for some health complications. In cases where both the husband and wife are positive, this fear takes a toll on both partners. Women who are detected during their ante-natal period however, receive free medication and treatment in Malaysia and the child is eligible for treatment until the age of eighteen. However, when the husband has to be on treatment as well, then the fear of financial constraint becomes a major issue which prevents the PLHIVs from seeking treatment. This is due to the financial implication on the household expenditure and socio-economic impact of the cost of treatment and care on the well-being of the family as a whole.

\section{2: $\quad$ Coping with internalised fear - the role of the CBO.}

The PLHIVs internalised fear needs to be handled foremost in order to enable the PLHIVs to take charge of their mental health and health behaviour. In general, the CBOs impart information, provide social support, assist to increase self-efficacy and motivate the PLHIVs to assume responsibility for change. Generally, the four outcomes targeted at the intervention practices include living life positively, increasing control on health behaviour, overcoming HIV/AIDS stigma and building an active participating community. In their effort 
to help PLHIVs to live life positively, the CBOs personnel focus particularly on increasing the self-efficacy of the PLHIVs. In order to restore the PLHIVs belief in their ability to live life positiively, the personnels focus on the HIV infection stage as well as the mode of infection. The PLHIVs who are in the earlier stage of detection generally experience greater emotional and psychological ordeal and this affects their self-esteem as well as their belief that they would be able to take control of their health compared to the PLHIVs who are already receiving HAART treatment. For this group of PLHIVs, the CBOs focus on giving hope for their future and reducing self-blame. The presence of their dependents and effects on family members will be focused particularly in counselling sessions if the PLHIVs do not take charge of controlling their health behaviour. The PLHIVs are also motivated to utilise the various financial assistance for medication and treatment provided by the Malaysian government which is not provided in some of the neighbouring countries. Such information is pertinent in giving hope for the PLHIVs to respond to their internalised fear.

The mode of infection on the other hand enables the CBOs personnel to focus on different topics of discussion during the counselling session. Women PLHIVs, for example are more accepting of fate, "karma" and "takdir" and religion becomes a strong source of motivation for them to take charge of their health condition. As for the male PLHIVs who have had multiple sex partners, service to other mankind and God is used as repentance as well as an opening to come out of their internalised fear. When the belief system is build upon the tenets of religious understanding, then intervention effort becomes much easier for the CBOs personnel particularly in reducing their internalised fear and increasing control on health behaviour. In particular, the CBOs help the PLHIVs to accept their seropositive status and learn to take control of their health condition as well as lead a healthy lifestyle. Peer group support session and regular meetings are held to motivate the PLHIVs to confront their internalised fear. Besides this, the PLHIVs are assisted to cope with stress and emotions caused by their new status and handle their fear in a more realistic manner by making the right decision and solving their problems more practically. The main focus is to adopt a healthy lifestyle in order to continue living with HIV infection with a positive outlook as there is hope to lead a normal life for a longer duration of life. Overcoming HIV/AIDS stigma and building active participating community however, takes a longer process and a different approach. Restoring the internalised fear alone may not be the answer to the PLHIVs to come forward to help overcome HIV/AIDS stigma. The PLHIVs are not able to counter the wider system in which the stigmatisation is constructed by the socio-cultural conviction particularly in the context of Malaysia. The PLHIVs are willing to adopt behaviour change for self improvement but need more effort to contest the society at large where the stigmatisation of HIV/AIDS is widespread.

Given the Malaysian socio-cultural construct however, the effort to fight stigma may be an arduous task, moreso when religion is misused as a weapon to suppress the rights and needs of the various groups of PLHIVs. Within the group however, the PLHIVs are willing to come forward and participate in peer-group sharing programmes and activities for support groups. However, it is noted that the women PLHIVs are more involved and supportive in joining the support groups compared to the homosexual groups and the men. This probably may be due to the fact that in Malaysia, the women PLHIVs are mostly portrayed as the "victims of HIV infection" and as such they are accepted with more compassion by the society compared to the men who are associated to behaviours that are considered to be against the norms of the society or linked to crime. The women too generally shoulder the burden of care of the family and children. That is why the society is more compassionate towards the PLHIV community. These factors also equally affect the building of an active participating community amongst the PLHIVs. Internalised fear helps to modify individual behaviour change but it does not unite the group to advocate their needs and rights in a more organised manner. Very few organisations are initiated by the PLHIVs themselves to fight for their rights until only recently where PLHIVs members are coming forward to build their own organisations in Malaysia. Usually, the PLHIVs depend highly on the CBOs to advocate their rights.

It is a pertinent aspect to note that in the context of HIV/AIDS issue in Malaysia, the two main factors that impinge on the empowerment process of the PLHIV community to confront their internalised fears are the environmental factor as well as the shortage of professional staffs in the organisations. Of particular concern encircling the environment factor is the issue of stigma that affects the PLHIVs community and the people in their surroundings. Although HIV/AIDS has been present for about a quarter century in Malaysia, yet it is difficult to draw out a clear framework to address either the issue of HIV/AIDS stigma and discrimination or in keeping a record of stigmatising incidence against PLHIVs to advocate the different PLHIV community's rights.

The issues of stigma and discrimination are in fact global challenges that affect PLWHAs empowerment in coping with internalized fear. Stigma and discrimination not only occurs in Malaysia, but is also common in other parts of the world, even as far as European Countries, Brazil, South Africa, Kenya, Thailand, India, Cambodia, China, Uganda, Indonesia and all parts of the world [7]. The shortage of professional staffs in the organisation too impinges the empowerment of PLHIVs to confront their internalised fear. This is because therapeutic counselling session needs professional training and guidance in order to sustain and monitor the intervention effort. While there are professional services available in Malaysia, the involvement 
of the professionals in the community-based organisation is limited. At the same time, the organisational personnel too are not able to cater totally to intervene at a professional level due to the multiple tasks of managing the organisation and working with limited number of staffs. Moreover, confronting the internalised fear among the PLHIVs in reality needs to be looked from the macro level whereby the stigmatisation of the pandemic contributes mainly to the presence of internalised fear among the PLHIVs. This of course, demands a revamp in changing the mindset of the society so that the stigmatisation of the pandemic does not impede the effort to assist PLHIVs to seek treatment as well as lead a healthy lifestyle.

Several questions arise in bringing about a change in the mindset of the society when we look at it at the micro level as well as the macro level of the society. Firstly, we need a clear understanding of how the Malaysian society interprets the issue of HIV in Malaysia. Does the Malaysian society view HIV pandemic as an act of ignorant behaviour, a malady of moral intolerance of unacceptable social norms or is the Malaysian society willing to accept the HIV spread as a health hazard which impinges on the positive growth of the nation's human development. If the issue of ignorant behaviour comes to the core, then we need to consider more innovative and practical educational and awareness initiatives to be disseminated to the various groups of society, stakeholders as well as policy makers. Besides, there will be a need to look at other peripheral issues that contributes to the ignorant behaviour. Likewise, if the HIV issue is seen as a malady of moral intolerance, then we need to define the morally accepted acts within the socio-cultural context of the society. This is definitely an arduous task as what may seem to be right to one may not be so for another, more so in a multirace and multi-cultural society setting. Finally, if the Malaysian society is willing to accept the HIV spread as a health hazard which threatens the nation's human development, then the question that comes to the fore is how prepared is the society to set aside their personal prejudice and stigmatisation of the pandemic and work towards containing the spread of the HIV infection. In short, how ready are we to embrace change in order to put aside moral questioning to HIV infection and treat it purely on a health issue? Can we at least educate the families to accept a HIV patient as someone who will need medical help in order to prolong a positive health? Given the socio cultural nuances of the Malaysian society, the effort to change the society's perception of HIV/AIDS stigma is definitely an arduous task which needs a long period of time to materialise. Such effort need to be complemented with clear policies, strong political wills as well as undivided societal support so that HIV infection will be seen as a health issue instead of a moral concern in the society. The unfailing effort by community-based organisations in helping the PLHIVs in confronting their internalised fear however, is indeed a Herculean effort which has earned a victory for the community-based organisations in empowering the PLHIVs in confronting their internalised fear.

\section{CONCLUSION}

The study shows that the PLHIV community experiences various types of internalised fear according to the diverse groups. Some of these fears are inevitable, particularly when financial matters arise; careers are at risk; family support fails or if the PLHIVs health condition deteriorates. However, a major contributory factor that evokes internalised fear among the PLHIVs is the stigmatisation of the pandemic where PLHIVs have a presupposed notion that they will succumb to a certain discriminatory practices in their surroundings. This presupposed notion is possibly learnt from previous experiences shared by other PLHIVs or through prejudicial views from a variety of source such as personal beliefs, societal norms, media reporting or social construction of the AIDS pandemic in Malaysia itself. As such, one pertinent aspect which needs to be given serious consideration in containing the spread of HIV is to focus on issues of stigma and discrimination that affects effective prevention of HIV/AIDS and subsequently reduce the fear among those living with HIV/AIDS. There are several recommendations to realize this notion.

Firstly, the society must accept the presence of HIV/AIDS as a health hazard to the nation. From the lowest level to the highest policy makers, acceptance of HIV/AIDS as a health issue should be accepted. Nonacceptance and denial will only leave an adverse effect on the economy and the nation building besides impeding all intervention and prevention effort. As such, highlighting and reframing HIV/AIDS as a health issue will need the cooperation of all parties, especially the media in its agenda setting about the issue. When the HIV/AIDS issue is seen as a health issue, then a great deal of stigmatising practices can be prevented as PLHIVs will be recognised as people who need psychosocial rehabilitation, health care and medical treatment. Such change of perception towards PLHIVs is necessary to mitigate the stigmatisation of the pandemic. Secondly, the PLHIVs community should be protected from discriminatory practices with the support of legislation and law that clearly defines elements and acts of stigma and discrimination against PLHIVs. The information about the law and legislation should also be disseminated to the PLHIVs in order to create awareness of their human rights and liberty. This effort should also be supplemented with the implementation of clear national policies which covers the PLHIV communities' basic human rights to respect, privacy and confidentiality as well as employees liberty to rightful employment and employment benefits besides preventive measures in workplace.

Likewise, the PLHIV community should also be protected from all forms of discriminatory practices; 
being it subtle or direct and all the children and family members of the PLHIVS should also be protected from any forms of discriminatory practices. The PLHIVs, on the other hand should also articulate their discredited experience through various channels or organisation as a mean to create awareness and educate the general public of their afflictions. This method of humanizing HIV/AIDS is quite rare in the Malaysian context, unlike in the west where Magic Johnson, Rock Hudson, Makgatho Mandela and many other well known people's HIV status was disclosed openly in order to create awareness and compassion as well as to eradicate stigmatisation of the HIV/AIDS issues. Such disclosure will enable to reduce the internalised fear among PLHIVs as well as invoke a compassion towards their family whom they have left behind. Besides humanizing HIV/AIDS, the PLHIVs also can organise and advocate their rights and lobby for their own concern. Insofar, there is already a coalition of PLHIV group in Malaysia that has come forward to provide social support for the PLHIVs but it will be a good effort if the organisation moves forward to advocate more strongly issues pertaining to stigma and discrimination among the PLHIVs too. Besides this effort, the support system that is established through the various networking with the community-based organisations, legal aid organisations, womens' group, gay community as well as other stakeholders should give due priority to issues on stigma and discrimination so that the PLHIVs community will not be segregated from the mainstream society. A holistic community involvement will then favourably allow the change of mindset amongst the public in accepting HIV/AIDS as a health problem. Although such effort takes time to materialise, the stigmatisation of HIV/AIDS issue in Malaysia has been present in the society long enough for the society to acclimatize itself for the benefit of the whole nation.

This study also insinuates the need for further research which may be carried out in a more comprehensive nature covering a bigger population of PLHIVs internalised fear as there may be untapped areas. A bigger sample representing the married group, unmarried group as well as the marginalised population of homosexual, commercial sex workers, children and the affected community will give clearer evidence-based situations that will enable appropriate actions to be undertaken. Such initiative will enable specific policies to be implemented to safeguard and protect the PLHIVs community. Likewise, it is necessary to examine the attitudes of the general population who inflict stigmatised practices towards the PLHIVs so that the elements of fear against the PLHIVs can be minimised through social campaigns and interpersonal approaches. When the issue of stigma and discrimination is seen from a varied perspective, then there can be a distinct effort to position HIV/AIDS-related stigma and discrimination within a broader social, cultural, political, and economic framework.

\section{Acknowledgements}

The authors wish to thank everyone involved in the study, particularly Professor Ismail Baba for his unfailing support, the PLHIVs who had given the trust to be interviewed and the support of all the organization's personnel for their cooperation throughout.

\section{REFERENCES}

[1]. Baba, Ismail. Psychosocial aspects of HIV/AIDS prevention. A look at CASP Penang. In Mohd Riji, Haliza \& Schweizer, Pataki K.J. HIV/AIDS - The realities, the challenges. Kuala Lumpur, Malaysia; 2002.

[2]. Rappaport.J Terms of empowerment/exemplars of prevention: Towards a theory of community psychology. American Journal of Community Psychology, 1987. 15(2), 121-148.

[3]. McArdle, J Community development. Resource manual for facilitators in community development. In Fleming, Mary Louise O'Connor and Parker, Elizabeth. Health promotion, principle and practice in the Australian context. Australia: Allen \& Unwin; 2001.

[4]. Kubler Ross, Elisabeth. On death and dying. MacMillan: New York; 1969.

[5]. Page and Nyakanda. How to live positively: facilitating community action in HIV/AIDS affected areas of 2003. Retrieved from: ebookbrowse.com/live-positively-cabl-pdf-d169903556.

[6]. Badri, Malik. The AIDS crisis: A natural product of modernity's sexual revolution. Kuala Lumpur. Malaysia: Madeena Books; 2000.

[7]. Singhal, Arvind and Roger, Everett. Combating AIDS: communication strategies in action. SAGE; 2003.

[8]. Goffman, Erwing. Stigma: Notes on the management of a spoiled identity. New York: Simon and Schuster; 1963.

[9]. Gostlin, L.O, Lazzarini, Z, AlexanderA, Brandt. A,M, Mayer, K.H \& Silverman D.C HIV testing, counselling and prophylaxia following sexual assault. Reproductive Health Matters. In Roziah Omar, Health-bridging the gap. Kuala Lumpur: University of Malaya Press; 1995.

[10]. Ministry of Health Malaysia and UNICEF Women and Girls. Confronting HIV and AIDS in Malaysia. Kuala Lumpur, Malaysia; 2008. 